\title{
Resumos dos trabalhos brasileiros apresentados no Congresso do Colésio Americano de Reumatologia (ACR) 2003
}

\section{Abstracts of the Brazilian studies presented at the American College of Rheumatology (ACR) 2003}

(ordem cronológica de apresentação)

Faria AC, Barcellos KSA, Andrade LEC: "Anti-ENA Antibody Longitudinal Oscillation in a 15-year Followup of a SLE" (Oscilação Longitudinal do Anticorpo Anti-ENA em Coorte de Pacientes com LES: Seguimento de 15 Anos). Arthritis Rheum 2003;48(9),S61. Instituição: UNIFESP, São Paulo, SP, Brasil.

Objetivo: Em oposição aos anti-DNA, os anticorpos antiENA (Sm, snRNP, Ro/SSA e SSB/La) são considerados razoavelmente estáveis ao longo do seguimento de pacientes com LES. Para verificar essa premissa, realizamos uma análise retrospectiva de determinações sucessivas de anticorpos anti-ENA e anti-DNA em um coorte de pacientes com LES durante um período de 15 anos. Métodos: Cento e trinta pacientes (93\% deles mulheres) foram selecionados retrospectivamente a partir dos registros do laboratório de Reumatologia. Todos os pacientes preencheram os critérios de LES do ACR e fizeram ao menos cinco testes de antiENA (imunodifusão dupla) e anti-dsDNA (IIF em Crithidia luciliae) entre 1987 e 2002. Quatro diferentes padrões de comportamento longitudinal foram considerados para cada anticorpo: 1) sempre ausente; 2) sempre presente; 3) ausente no diagnóstico com posterior soroconversão; e 4) presente no diagnóstico, mas com testes negativos subseqüentes. Resultados: O número médio de testes por paciente foi de 17 (5 a 76) em um período médio de 8 anos ( 5 a 15 anos). Anti-Ro/SSA foi positivo em 61 pacientes (47\%), antisnRNP em 47 (36\%), anti-DNA em 42 (32\%), anti-Sm em $30(23 \%)$ e anti-SSB/La em 9 (7\%). Somente uma porcentagem limitada desses pacientes apresentou testes positivos durante todo o seguimento (52\% anti-Ro/SSA; 38\% antisnRNP; $17 \%$ anti-Sm, 11\% anti-SSB/La e 9\% anti-DNA). Os pacientes remanescentes apresentaram comportamentos oscilatórios (padrões 3 e 4). Entre aqueles com teste positivo no diagnóstico houve uma diminuição progressiva do número de pacientes que permaneceram positivos ao longo dos anos: a freqüência dos pacientes que permaneceram positivos entre o $2 .^{\circ}$ e o $4 .^{\circ}$ anos e entre o $5 .^{\circ}$ e o $10 .^{\circ}$ anos de seguimento diminuiu progressivamente para os anticorpos anti-SSB/La (40\% e 20\%), anti-DNA (39\% e $25 \%)$, e anti-Sm (50\% e 27\%). Inversamente, um grupo de pacientes apresentou testes negativos no diagnóstico com posterior soroconversão (padrão 3), abrangendo 56\% dos pacientes anti-SSB/La, 33\% dos anti-DNA, 26\% dos antiSm, 21\% dos anti-snRNP e 15\% dos anti-Ro/SSA. A soroconversão ocorreu em um intervalo médio de 2 (anti-Ro/ SSA-SSB/La), 2,8 (anti-snRNP), 3 (anti-Sm) e 3,6 (antiDNA) anos após o diagnóstico. A mais freqüente associação de anticorpos no mesmo paciente ocorreu para anticorpos anti-Sm e anti-snRNP (29 pacientes, $22 \%$ do total). Entre os últimos, 12 (41\%) pacientes apresentaram resultados do teste idênticos para ambos os anticorpos durante todo o seguimento. Os quatro modelos de comportamento longitudinal foram observados para todos os anticorpos. A positividade permanente (padrão 2) foi observada mais freqüentemente para anti-Ro/SSA e anti-snRNP em comparação com anticorpos anti-Sm e anti-DNA (qui-quadrado, $\mathrm{p}<0,05$ ). Padrões oscilatórios (padrões 3 e 4 ) foram mais freqüentemente observados para anticorpos anti-Sm e antiDNA em comparação com anticorpos anti-Ro/SSA e anticorpos anti-snRNP $(\mathrm{p}<0,05)$. A freqüência do modelo de comportamento não diferiu entre anticorpos anti-snRNP e anti-Ro/SSA $(p=0,15)$ nem entre anticorpos anti-DNA e anti-Sm $(p=0,29)$. Conclusões: A análise retrospectiva de 130 pacientes com LES representou alta freqüência de oscilação para anticorpos anti-DNA e anti-ENA. Anticorpos anti-Sm e anti-DNA apresentaram comportamento oscilatório alto, e os anticorpos anti-Ro/SSA e anti-snRNP apresentaram comportamento mais estável. 
Schecher SJ ${ }^{(1)}$, Sundel ${ }^{(2)}$, Oliveira SK ${ }^{(3)}$, Huttenlocher $\mathrm{A}^{(4)}$, Onel $\mathrm{K}^{(1)}$, Lehman TJA ${ }^{(1)}$ : "Thalidomide for severes systemic onset JIA" (Talidomida para AIJ sistêmica severa). Arthritis Rheum 2003;48(9),S92. Instituições: 1. Hospital for Special Surgery, New York, NY; 2. Children's Hospital, Boston, MA; 3. Universidade Federal do Rio de Janeiro, Rio de Janeiro; Brasil, 4. Universidade de Wiscosin Medical School, Madison, WI.

Relatos prévios documentaram a eficácia da terapia com talidomida em crianças com AIJ sistêmica dependente de corticosteróides em um único centro. Este relato documenta a experiência estendida a 13 pacientes com AIJ dependente de corticosteróides em 4 diferentes centros. Todas as crianças tinham AIJ sistêmica severa dependente de corticosteróides ao entrarem no estudo. Todos receberam talidomida 3 - 5 $\mathrm{mg} / \mathrm{kg} /$ dia. A dose de prednisona, velocidade de hemossedimentação, hemoglobina, leucócitos e número de articulações ativas foram registradas antes do tratamento e após 6, 12, e 24 meses.

Estes dados, provenientes de múltiplos investigadores de diferentes centros, demonstram que a talidomida é uma terapia eficaz na AIJ sistêmica resistente a corticosteróide. Seu uso está associado a uma dramática melhora da hemoglobina, velocidade de hemossedimentação e número de articulações ativas, com uma redução simultânea da dose de prednisona após 6 meses e 2 anos de acompanhamento.

\begin{tabular}{|c|c|c|c|c|}
\hline & $\begin{array}{c}\text { Pré-tto } \\
n=13\end{array}$ & $\begin{array}{c}6 \text { meses } \\
n=13\end{array}$ & $\begin{array}{c}12 \text { meses } \\
n=5\end{array}$ & $\begin{array}{c}24 \text { meses } \\
n=5\end{array}$ \\
\hline Leucócitos/mm³ & $11.8 \pm 1.76$ & $9.3 \pm 1.6$ & $6.8 \pm 0.9$ & $7.0 \pm 1.9$ \\
\hline $\mathrm{Hb}(\mathrm{gm} \%)$ & $10,4 \pm 0,53$ & $\begin{array}{c}12,0 \pm 0,4 \\
p<0,005\end{array}$ & $\begin{array}{c}11,5 \pm 0,53 \\
p<0,05\end{array}$ & $\begin{array}{c}11,1 \pm 0,9 \\
p<0,05\end{array}$ \\
\hline VHS & $54,4 \pm 8,2$ & $\begin{array}{c}23,1 \pm 6,3 \\
p<0,0005\end{array}$ & $\begin{array}{c}19,4 \pm 6,7 \\
p<0,01\end{array}$ & $\begin{array}{l}31 \pm 15 \\
p<0,025\end{array}$ \\
\hline N. ${ }^{\circ}$ articulações & $19,1 \pm 8,2$ & $6,8 \pm 2,7$ & $9,2 \pm 4,1$ & $9,2 \pm 4,1$ \\
\hline Prednisona mg & $14,8 \pm 3,8$ & $5,1 \pm 2,3$ & $6,5 \pm 5$ & $3,0 \pm 2,0$ \\
\hline
\end{tabular}

Azevedo PM, Hayata ALS, Borba EF, Assad RL, Kochen JAL, Goldenstein-Schainberg C: "High Frequency of Dyslipoproteinemia as a Risk Factor for Coronary Artery Disease (CAD) In Juvenile Idiopathic Arthritis (JIA)" (Alta Freqüência de Dislipoproteinemia como Fator de Risco para Doença Arterial Coronária [DAC] na Artrite Idiopática Juvenil [AIJ]). Arthritis Rheum 2003;48(9),S97. Instituição: USP, São Paulo, Brasil.

A dislipoproteinemia (DL) é um importante fator de risco para DAC. Na artrite reumatóide (AR) em adultos observou-se redução da expectativa de vida, atribuída principalmente à morte cardiovascular (30\% a 40\%). Com relação a crianças com AIJ, os dados sobre o perfil lipídico são limitados e inconclusivos. Portanto, estudamos a prevalência de DL em um coorte de pacientes com AIJ. Pacientes e Métodos: Foram avaliados 40 pacientes com AIJ, de acordo com os critérios da ILAR revisados em 1997, regularmente acompanhados em nossa unidade. Pacientes com diabetes, disfunção tireoidiana, proteinúria nefrótica, fumantes e em terapia hormonal ou diurética foram sistematicamente excluídos do estudo. O perfil lipídico no plasma foi determinado após 12 horas de jejum. A atividade da doença foi arbitrariamente estabelecida como a presença de sinovite em uma ou mais articulações associadas a proteínas de fase aguda elevadas (VHS, PCR). Os níveis de risco de lipoproteína para DAC foram definidos de acordo com o Programa 
Nacional de Educação sobre o Colesterol. Comparações estatísticas foram realizadas utilizando-se o teste nãoparamétrico de Mann-Whitney e um valor de $\mathrm{p}<0,05$ foi considerado significativo. O risco relativo de DL segundo a atividade da doença e a duração desta foi estimado com odds ratio (OR) e intervalo de confiança de 95\% (IC 95\%) utilizando-se regressão logística incondicional. Resultados: Foram avaliados 40 pacientes com AIJ (6 casos de sistêmica, 28 de poliarticular e 6 de oligoarticular) com média de idade $=18,9 \pm 5,4$ anos (faixa de 5 a 29 anos) e duração média da doença $=8,6 \pm 5,2$ anos (faixa de 1 a 25 anos). Doze pacientes estavam sob terapia com CE $(\leq 5 \mathrm{mg} / \mathrm{kg} /$ dia) e 23/40 (58\%) com AIJ ativa. Notavelmente, todos os pacientes, exceto um, 39/40 (97,5\%), apresentaram níveis de risco médio/alto de HDL, embora a metade deles estivesse tomando cloroquina (Tabela). Quase um quarto dos pacientes, 9/40 (23\%), apresentou níveis de risco médio/ alto de TC e 6/40 (15\%) apresentaram níveis de LDL médio/ alto. Níveis de risco médio/alto para DAC nesses pacientes foram associados a maior duração da doença $\geq 10$ anos para $\mathrm{TC}(\mathrm{OR}=1,96 ;$ IC $95 \%=0,3)$ e $\mathrm{TG}(\mathrm{OR}=2,4 ; \mathrm{IC}$ $95 \%=0,4)$, mas não para LDL $(\mathrm{OR}=1,05 ;$ IC $95 \%=0,1)$ ou HDL (OR $=0$; IC 95\% = 0,3).

Conclusão: A alta freqüência de níveis de risco médio/ alto de DL para DAC em pacientes com AIJ reforça a necessidade de medidas preventivas a fim de diminuir os possíveis efeitos deletérios desse distúrbio. A correlação positiva dessa alteração com a duração da doença enfatiza ainda mais a relevância dessa intervenção levando-se em conta a longa expectativa de vida desses pacientes.

TABELA

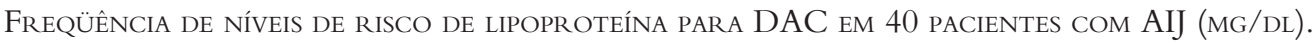

\begin{tabular}{lcccccccc}
\hline Risco & TC & LDL & HDL & TG & & nol \\
& nivel & N (\%) & nivel & N (\%) & nivel & N (\%) & nivel & N (\%) \\
\hline Baixo & $<170$ & $31(77,5)$ & $<110$ & $34(85)$ & $>71$ & $1(2,5)$ & $<130$ & $35(87,5)$ \\
Médio & $170-199$ & $7(17,5)$ & $110-129$ & $2(5)$ & $36-71$ & $32(80)$ & \\
Alto & $\geq 200$ & $2(5)$ & $\geq 130$ & $4(10)$ & $\leq 35$ & $7(17,5)$ & $\geq 130$ & $5(12,5)$ \\
\hline
\end{tabular}

Curtis $\mathrm{SP}^{(1)}$, Losada $\mathrm{B}^{(2)}$, Bosi-Ferraz $\mathrm{M}^{(3)}$, Saenz $\mathrm{R}^{(4)}$, Miller ER ${ }^{(1)}$, Ray $\mathrm{P}^{(1)}$, Reicin $A S^{(1)}$ : "Etoricoxib and Diclofenac Demonstrate Similar Efficacy in a 174-Week Randomized Study of Rheumatoid Arthritis Patients" (Etoricoxib e Diclofenaco Demonstram Eficácia Similar em um Estudo Randomizado de 174 Semanas Envolvendo Pacientes com Artrite Reumatóide). Arthritis Rheum 2003;48(9), S124-5. Instituições: 1. Merck Research Laboratories, Rahway, NJ; 2. Clínica Atías, Caracas, Venezuela; 3. UNIFESP/EPM, São Paulo, Brasil; 4. Centro de Especialidades Médicas Dr. Heredia, Costa Rica.

Objetivo: O etoricoxib, um inibidor seletivo da COX-2, demonstrou ser eficaz na artrite reumatóide (AR). Anteriormente à participação em dois estudos consecutivos de Extensão, os pacientes com AR participaram de um estudo de Base duplo-cego de 52 semanas (um período de 8 semanas placebo-controlado seguido por um período de 44 semanas comparador-ativo-controlado). O estudo Base demonstrou que o etoricoxib $90 \mathrm{mg}$ (E90) e $120 \mathrm{mg}$ (E120) uma vez por dia foram mais eficazes que o etoricoxib $10 \mathrm{mg}$ (E10) e $60 \mathrm{mg}$ (E60) e placebo (PBO) durante 8 semanas; e similar em termos de eficácia ao diclofenaco $50 \mathrm{mg}$ três vezes por dia (D150) durante as 44 semanas subseqüentes. Métodos:
Os pacientes forneceram consentimento na 52. ${ }^{a}$ semana (e na 104. ${ }^{a}$ semana) para ingressar no primeiro (e segundo) estudo de Extensão, duplo-cego, com comparador ativo (diclofenaco), placebo controlado x comparador-ativocontrolado, desenhado para avaliar a manutenção a longo prazo da eficácia e da tolerabilidade do etoricoxib por 52 (e 70) semanas adicionais. As avaliações foram feitas periodicamente de 3 a 4 meses. Os endpoints primários de eficácia incluíram a Avaliação da Dor pelo Paciente (EAr) e as Avaliações Globais da Atividade da Doença pelo Investigador. As avaliações de segurança incluíram avaliações física e laboratorial e experiências adversas (EAs). 
Resultados: Dos 197, 85 e 93 pacientes ingressaram na primeira Extensão nos grupos E90, E120 e D150, respectivamente; 127, 67 e 70 pacientes ingressaram na segunda Extensão, respectivamente; e 104, 61 e 60 pacientes com-

GRUPOS DE TRATAMENTO

\begin{tabular}{lcc}
\hline Estudo de base & \multicolumn{2}{c}{ Extensões } \\
\hline Baseline 8. ${ }^{\text {a Semana }}$ & Semanas 8 a 52 & Semanas 52 a 174 \\
\hline Placebo & D150 $(50 \%)$ & D150 \\
& E60 $(50 \%)$ & E90 \\
Etoricoxib $10 \mathrm{mg}$ & D150 $(50 \%)$ & D150 \\
Etoricoxib $60 \mathrm{mg}$ & E60 $(50 \%)$ & E90 \\
& E60 $(50 \%)$ & E90 \\
Etoricoxib $90 \mathrm{mg}$ & E90 $(50 \%)$ & E90 \\
Etoricoxib $120 \mathrm{mg}$ & E90 $(100 \%)$ & E90 \\
\hline
\end{tabular}

pletaram as 174 semanas, respectivamente. A eficácia nos grupos E90, E120 e D150 durante as semanas 52 a 174 foi mantida em um nível relativamente constante para todos os endpoints primários, similares em magnitude àqueles relatados no estudo Base (semanas 8 a 52).

E90, E120 e D150 foram geralmente bem tolerados durante as Extensões (semanas 52 a 174). Não houve achados novos ou exclusivos de segurança com exposição a longo prazo de E90, E120 e D150 até as 174 semanas. Conclusões: Etoricoxib 90 e $120 \mathrm{mg}$ uma vez por dia fornecem eficácia a longo prazo similar ao diclofenaco 50 mg três vezes por dia quando administrado a pacientes com AR por até 174 semanas. Todos os grupos que continuaram a terapia após aproximadamente um ano de tratamento demonstraram manter a eficácia. Etoricoxib 90 e 120 mg e diclofenaco são geralmente seguros e bem tolerados por pacientes com AR até 174 semanas de tratamento.

Avaliação da Dor pelo Paciente (100 mm EAV)

\begin{tabular}{lllll}
\hline & \multicolumn{2}{c}{ Semanas 8 a 52} & \multicolumn{2}{c}{ Semanas 52 a 174 } \\
& N & Faixa de Redução Média* & N & Faixa de Redução Média * \\
\hline Etoricoxib $90 \mathrm{mg}$ & 190 & 34,7 a 37,5 & 197 & 34,7 a 39,6 \\
Etoricoxib $120 \mathrm{mg}$ & 110 & 31,7 a 37,5 & 85 & 36,2 a 40,6 \\
Diclofenaco $150 \mathrm{mg}$ & 130 & 32,4 a 40,7 & 93 & 38,2 a 43,2 \\
\hline
\end{tabular}

* A faixa são os valores mínimo e máximo para média a cada visita

EAV = Escala Analógica Visual

Turrini-Filho JR, Kuruma KAM, Bertola C, Pedalini MEB, Borba EF, Bonfá E: "Lack Of Child Ototoxicity By Limiar Tonal Audiometry (LTA) After Chloroquine Gestacional Use In Systemic Lupus Erythematosus" (Ausência de Ototoxicidade em Crianças Definida por Audiometria Tonal Limiar [ATL] após Uso Gestacional de Cloroquina no Lúpus Eritematoso Sistêmico). Arthritis Rheum 2003;48(9), S180. Instituição: Divisão de Reumatologia, USP, São Paulo, SP, Brasil.

O objetivo do estudo foi avaliar ototoxicidade dos antimaláricos em crianças após sua utilização durante o período gestacional de pacientes com LES. Avaliamos prospectivamente 19 crianças de mais de 4 anos de idade, nascidas de mães com LES, sem história anterior de otite recorrente, trauma acústico e infecções. Nove delas foram expostas ao difosfato de cloroquina (DFC) durante a gestação (dose cumulativa média de 34,166 $\pm 23,750 \mathrm{mg}$ ), enquanto dez nasceram de mães que não ingeriram essa droga 6 meses antes da concepção ou em qualquer outro momento da gravidez (controles). Todas as 9 mães do grupo-DFC foram expostas a essa droga, pelo menos, no primeiro trimestre de gravidez e 5 delas, durante todo o período gestacional. A audiometria tonal limiar (ATL) foi realizada em todas as crianças por um especialista sem conhecimento do tratamento. A avaliação audiométrica foi avaliada por média de tom puro em alta $(2.000,3.000,4.000$ e $8.000 \mathrm{~Hz})$ e baixa $(250,500$ e $1.000 \mathrm{~Hz})$ frequiências e comparada entre os grupos para identificar perda de audição sensorineural e condutiva. Resultados: Não houve diferença significativa com relação à duração da gestação $(35,8 \pm 2,1$ versus $36,8 \pm 1,8$ meses, $\mathrm{p}=0,36)$ e à idade $(7,55 \pm 4,36$ versus $12,3 \pm 7,22$ anos, $\mathrm{p}=0,10)$ entre os grupos DFC e controle. Em todas as crianças, obteve-se história de aquisição normal da habilidade de 
comunicação, bem como performance escolar condizente com a idade. As avaliações audiométricas estiveram dentro dos níveis normais em ambos os grupos. Conforme esperado, a média

MÉdia de ALTA FREQÜÊNCIA PARA CADA OUVIDO DE CRIANÇAS EXPOSTAS A DFC E NÃO-EXPOSTAS A ESSA DROGA (CONTROLES) CONFORME MEDIÇÃO PELA AUDIOMETRIA TONAL LIMIAR

\begin{tabular}{llll}
\hline Ouvido & DFC $(n=9)$ & Controles $(n=10)$ & $p$ \\
\hline Direito & $9,0 \pm 4,9$ & $5,6 \pm 4,0$ & 0,12 \\
Esquerdo & $9,2 \pm 4,5$ & $8,1 \pm 5,5$ & 0,63 \\
\hline
\end{tabular}

Valores expressos em média $\pm \mathrm{DP}$, em dBHL de audição dos ouvidos esquerdo e direito em baixas freqüências foi similar nos grupos DFC e controle $(\mathrm{p}=0,87 \mathrm{e}$ $\mathrm{p}=0,68$ respectivamente). Notavelmente, a medição de alta freqüência, normalmente associada à perda de audição induzida por drogas, foi idêntica em cada ouvido em ambos os grupos $(\mathrm{p}=0,12$ e $\mathrm{p}=0,63)$.

Conclusões: Os antimaláricos não parecem induzir ototoxicidade clínica ou subclínica em crianças com exposição in utero conforme determinado pela ATL, o que reforça sua utilização segura durante a gravidez de pacientes com lúpus.

Appenzeller S, Cendes F, Costallat LTL: "Epileptic seizures in Systemic Lupus Erythematosus" (Anticorpos Antifosfolípides e Acidente Vascular Cerebral associados à Epilepsia no LES). Arthritis Rheum 2003;48(9),S182. Instituição: Departamento de Clínica Médica e Neurologia da UNICAMP, Campinas, SP, Brasil.

Objetivo: Avaliar a freqüência de crises epilépticas em pacientes com lúpus eritematoso sistêmico (LES) e determinar fatores clínicos e laboratoriais associados a sua ocorrência. Pacientes e Métodos: Foram incluídos 519 pacientes com quatro ou mais critérios para LES (CAR, 1982), seguidos periodicamente no ambulatório de janeiro de 1974 a dezembro de 2001. Foram excluídos pacientes com crises epilépticas secundárias a infecções do sistema nervoso central, hipertensão arterial sistêmica, distúrbio metabólico e hipóxia. As associações entre crises epilépticas e as variáveis do estudo foram feitas através de regressão multivariada com processo de seleção "stepwise". Resultados: Desta coorte foram identificados 60 $(11,6 \%)$ pacientes com crises epilépticas. Crises epilépticas foram identificadas em 19 (29\%) pacientes no momento do diagnóstico e em 58 (63\%) durante a evolução. Crises únicas foram observadas em 58 de 65 (89\%) pacientes. Ao diagnóstico, as crises estavam associadas à presença de acidente vascular cerebral (AVC) $(p=0,0004 ; \mathrm{OR}=10,36 ; 95 \% \mathrm{CI}=2,8 ; 38,2) \mathrm{e}$ à presença de anticorpos antifosfolípides $(\mathrm{p}=0,0013 ; \mathrm{OR}=6,69$; $95 \% \mathrm{CI}=2,1 ; 21,4)$. Durante a evolução do LES, as crises epilépticas estavam associadas à presença de nefrite $(\mathrm{p}=0,001$; $\mathrm{OR}=3,2 ; 95 \% \mathrm{CI}=1,6 ; 6,5)$, anticorpos antifosfolípides e crises epilépticas agudas ao diagnóstico $(p=0,0001 ; O R=8,27$; $95 \% \mathrm{CI}=2,9 ; 23,3)$. Crises recorrentes foram observadas em 7 $(10.8 \%)$ pacientes e todos estes pacientes apresentaram a síndrome do anticorpo antifosfolípide. Conclusão: A presença de anticorpos antifosfolípides estava associada à ocorrência de crises epilépticas agudas e recorrentes. AVC foi outra manifestação do SNC associada à crises epilépticas agudas. Pacientes com LES e crises epilépticas devem ser cuidadosamente investigados quanto à presença de AVC e anticorpos antifosfolípides.

Braga Jr JWR, Pinheiro MM, Neves RMS, Heymann RE, Carvalho AB, Szejnfeld VL: "Prevalence of Osteoporotic Fractures in Long-term Kidney Transplant Patients with Preserved Renal Function" (Prevalência de Fraturas Osteoporóticas em Pacientes Submetidos a Transplante Renal Há Mais de Três Anos com Função Renal Preservada). Arthritis Rheum 2003;48(9), S300. Instituição: UNIFESP, São Paulo, SP, Brasil.

A insuficiência renal terminal está associada à diversas alterações do metabolismo ósseo e mineral, bem como elevado risco de fraturas. O transplante renal pode reverter muitas anormalidades metabólicas, entretanto a doença óssea pode persistir por longos períodos ocasionando baixa densidade óssea e fraturas. Neste estudo, os autores avaliaram 
a prevalência de baixa densidade óssea e fraturas por fragilidade em 191 pacientes submetidos ao transplante renal há mais de 3 anos, com função renal preservada (creatinina sérica menor que $2,5 \mathrm{mg} / \mathrm{dl}$ ) e determinaram os principais fatores de risco associados às fraturas por osteoporose nessa população. Os fatores de risco clínicos estudados incluíam etiologia da insuficiência renal, duração da diálise no prétransplante, tempo de transplante e de menopausa, diabetes mellitus, tipo de doador, dose cumulativa de corticosteróides, ciclosporina e azatioprina. A densidade mineral óssea e risco de fratura foram feitos por meio de DXA (Lunar, DPX) na coluna lombar e fêmur proximal e a ultra-sonometria óssea de calcâneo (BUA, SOS e índice stiffness). Fratura vertebral foi confirmada por análise radiológica e definida pelo método de Eastell. Quarenta e seis pacientes (24\%) relataram história prévia de fratura vertebral (29/46) ou apendicular (17/46) após o transplante renal. Foi observada osteoporose em 3\% a 13\% dos pacientes e osteopenia em 30\% a 37\%, dependendo do sexo e região examinada, de acordo com os critérios da OMS. Mulheres apresentaram mais fraturas que os homens. Os principais preditores de fratura em mulheres foram diabetes mellitus, tempo de menopausa, altas doses de corticosteróides, baixa densidade óssea do fêmur e reduzidos valores do índice stiffness. Nos homens, encontramos o tempo de diálise, baixa densidade óssea da coluna lombar e baixos valores do índice stiffness. Nossos resultados demonstram alta prevalência de baixa densidade óssea e fraturas por fragilidade em pacientes submetidos ao transplante renal e apontam a necessidade de intervenção específica para prevenir osteoporose nessa população. Mulheres na pós-menopausa e diabéticos apresentam maior risco para fratura no período pós-transplante renal.

Assis MR, Silva LE, Alves A, Pessanha AP, Valim V, Feldman D, Barros Neto TL, Natour J: "'Deep Water Running' to Treat Fibromyalgia - an Aerobic Training in a Swimming Pool: a Randomized, Controlled Study" (Ensaio Controlado Aleatório para Tratamento da Fibromialgia com Deep Water Running - um Exercício Aeróbio em Piscina). Arthritis Rheum 2003;48(9), S303. Instituição: Setor de Reabilitação e Coluna Vertebral da Disciplina de Reumatologia e Centro de Medicina e Atividade Física e Esportiva da UNIFESP, São Paulo, SP, Brasil.

Introdução: $\mathrm{O}$ exercício aeróbico mostrou-se eficaz no tratamento da fibromialgia (FM). Embora a hidroterapia pareça ser útil na melhora dos sintomas da FM, um programa aquático independente prescrito com uma rigorosa avaliação fisiológica ainda não foi estudado. Objetivo: Comparar os efeitos do exercício aeróbio no meio aquático com o exercício em solo em mulheres com fibromialgia. Comparar o condicionamento físico e os efeitos adversos. Correlacionar melhora clínica com melhora de aptidão fisica. Material e métodos: Sessenta mulheres sedentárias com critérios para fibromialgia pelo ACR, com idade entre 18 e 60 anos, foram separadas por sorteio em dois grupos: corrida dentro d'água com cinto flutuador (deep water running - DWR) e exercício em solo (SOLO). O grupo DWR exercitou-se em piscina aquecida a $29^{\circ} \mathrm{C}$ e o grupo SOLO realizou caminhada ou corrida em um parque municipal. As pacientes treinaram por 15 semanas no seu limiar anaeróbio determinado por um teste ergoespirométrico. Os treinos foram monitorizados com freqüencímetros e supervisionados por fisioterapeutas. Escala visual analógica de dor (EVA), questionário de impacto da fibromialgia (FIQ), inventário Beck de depressão, questionário de qualidade de vida SF-36 e escala visual de melhora global (EVAM) foram aplicados à admissão no estudo, após 8 e 15 semanas. A análise estatística foi por intenção de tratamento (considerou todos os indivíduos) e incluiu teste T, ANOVA, qui-quadrado, Pearson e Spearman. Resultados: Houve 4 perdas em cada grupo. Ambos os grupos melhoraram na 15. ${ }^{a}$ semana quando comparados com a linha de base. Ambos os grupos melhoraram em 36\% a média da EVA. Na EVAM, 40\% do DWR e 30\% do SOLO se declararam "muito melhor" após o tratamento. No DWR, o escore total do FIQ e seu componente "depressão" melhoraram mais rapidamente (8 semanas) e também foram melhores que o SOLO na 15. ${ }^{a}$ semana $(\mathrm{p}<0,05)$. No DWR, houve melhora intragrupo no domínio aspectos emocionais do SF36 $(p=0,006)$, mas isso não ocorreu no SOLO. Não houve diferenças entre grupos nos outros resultados, incluindo o pico de consumo de oxigênio e o número de indivíduos que apresentaram intercorrências durante o estudo. Conclusões: $\mathrm{O}$ tratamento de fibromialgia com a DWR foi efetivo e comparável ao condicionamento aeróbio em solo. A DWR mostrou-se superior ao treinamento por caminhada ou corrida pelo FIQ total e por alguns itens de avaliação psicoafetiva. A melhora da dor e o ganho de condicionamento fisico foram semelhantes nos dois grupos. Não houve diferença significativa entre os grupos com 
relação a intercorrências. A melhora de sintomas não se correlacionou com a melhora no desempenho físico ao final do estudo. A corrida dentro d'água com cinto flutuador mostrou-se uma opção segura para o tratamento de pacientes com fibromialgia e poderia ser empregada em pacientes com dificuldade ou incapacidade para se exercitar em solo.

Baldin $\mathrm{GR}^{(1)}$, Andrade PA ${ }^{(1)}$, Alqualo-Costa $\mathrm{R}^{(1)}$, Gallinaro $\mathrm{AL}^{(2)}$, Lage $\mathrm{LV}^{(3)}$ : "Assessment of Sexual Activity in Patients with Fibromyalgia - A Controlled Study" (Avaliação da Atividade Sexual em Pacientes com Fibromialgia - Um Estudo Controlado). Arthritis Rheum 2003;48(9),S305. Instituições: 1. Disciplina de Fisioterapia, Universidade Cidade de São Paulo (UNICID), São Paulo, SP, Brasil; 2. UNICID, São Paulo, SP, Brasil; 3. Divisão de Reumatologia, USP, São Paulo, SP, Brasil.

A dor é uma questão primordial na síndrome da fibromialgia (SFM), haja vista que os músculos que doem sobre pressão podem sofrer um agravamento durante as relações sexuais, levando a problemas notáveis no que tange ao relacionamento sexual. Além disso, a SFM pode coincidir com problemas emocionais, tais como depressão, autoimagem negativa, ansiedade ou medo de rejeição, o que torna o problema ainda maior. Adicionalmente, as medicações antidepressivas podem agravar ainda mais o problema, interferindo nas funções e no desejo sexuais. No entanto, as funções sexuais de pacientes com fibromialgia foram raramente descritas na literatura. Objetivos: Comparar a qualidade da atividade sexual em pacientes com SFM com aquela de controles saudáveis. Pacientes e Métodos: Cinqüenta mulheres com vida sexual ativa que preenchiam os critérios do ACR para fibromialgia e 50 mulheres saudáveis foram convidadas para participar do estudo. Todas foram chamadas para preencher um questionário estruturado com perguntas relacionadas com sua vida sexual, inclusive dor e cansaço durante o intercurso sexual, iniciativa para o sexo, freqüência de intercurso sexual durante o último mês e grau de satisfação com sua vida sexual atual. Resultados: A média de idade no grupo-SFM foi de $44 \pm 9$ anos e de $40 \pm 9$ anos no grupo-controle $(\mathrm{p}=0,096) ; 64 \%$ das pacientes com SFM e 80\% das mulheres do grupo-controle tiveram relacionamentos sexuais regulares durante os últimos seis meses $(\mathrm{p}=\mathrm{n} . \mathrm{s})$. Pacientes com SFM estavam menos satisfeitas com sua vida sexual atual que as mulheres do grupo-controle ( $55 \%$ versus $21 \% ; \mathrm{OR}=4,8 ; \mathrm{p}<0,0001)$. As pacientes com SFM tendem a apresentar mais dor durante o intercurso sexual que as mulheres do grupo-controle ( $74 \%$ versus $36 \%$; OR $=5$; $\mathrm{p}<0,0001)$. Entre todas as mulheres que referiam sentir dor durante o intercurso sexual, as pacientes com FMS mostraram menos satisfação sexual que as mulheres do grupocontrole $(40 \%$ versus $61 \%$; OR $=2,23 ; p=0,008)$. Pacientes com SFM também apresentaram mais cansaço durante as relações sexuais que as mulheres do grupo-controle $(73 \%$ versus $37 \%$; $\mathrm{OR}=4,6 ; \mathrm{p}<0,0001$ ), além de menor tendência a iniciar a relação sexual que as pacientes do grupo-controle que referiram dor durante o coito ( $73 \%$ versus $50 \%$; $\mathrm{OR}=0,33 ; \mathrm{p}=0,0004)$. Não houve diferença significativa entre os dois grupos com relação à duração ou à freqüência de relações sexuais. Conclusão: A sexualidade é amplamente prejudicada nas pacientes com FM em comparação com as controles saudáveis. Este achado reforça a necessidade de uma abordagem multidisciplinar dessa doença a fim de oferecer uma melhor qualidade de vida a essas pacientes.

Kochen JAL, Andrade DC, Souza SCM, Takayama L, Borges CTL, Aldrighi JM, Pereira RMR: "Bone Loss and Fractures in Patients with Idiopathic Inflammatory Myopathies. The Role of Steroids, Disease Duration and Age" (Perda Óssea e Fraturas em Pacientes com Miopatias Inflamatórias Idiopáticas. Papel dos Esteróides, Duração da Doença e Idade). Arthritis Rheum 2003;48(9),S310. Instituição: Divisão de Reumatologia, USP, São Paulo, Brasil.

A polimiosite e a dermatomiosite são miopatias inflamatórias idiopáticas (MII) incomuns que podem estar associadas à perda óssea. Agentes terapêuticos tradicionais que afetam o metabolismo ósseo, principalmente os corticosteróides (CE), são utilizados extensivamente para tratamentos agudo e de longo prazo. Entretanto, sabe-se pouco sobre o impacto 
da doença e sobre os efeitos dos glicocorticóides no metabolismo ósseo nesse grupo de pacientes. Métodos: Foram estudadas 41 mulheres com MII e 262 mulheres saudáveis, pareadas por idade ( 23 a 74 anos, média de idade: $51,4 \pm 13,5$ versus $54,0 \pm 10,0)$. As características da doença, história de fraturas anteriores e detalhes relativos ao uso de CE foram obtidos por meio de questionário, registros médicos e entrevista com a paciente. Todas as pacientes receberam CE e 76,9\% utilizavam-no naquele momento. A dose cumulativa média de CE (equivalente à prednisona) foi de $35,23 \pm 33,70 \mathrm{~g}$. A doença em atividade foi observada em $29 \%$ das pacientes com MII, e a média de duração da doença foi de 9,96 \pm 9,5 anos. A densidade mineral óssea (DMO) foi medida por densitometria óssea de dupla emissão com fonte de raios X (DXA), densitometria óssea (Hologic QDR 2000) na coluna lombar e colo femoral. Resultados: Não foi encontrada diferença na altura, no peso, no índice de massa corpórea (IMC) e no status de menopausa entre os dois grupos. Observou-se diminuição na densidade mineral óssea de pacientes com MII em comparação com as controles saudáveis na coluna lombar $(0,906 \pm 0,137$ versus $0,956 \pm$ $\left.0,145 \mathrm{~g} / \mathrm{cm}^{2}, \mathrm{p}=0,04\right)$ e colo femoral $(0,731 \pm 0,121$ versus $\left.0,775 \pm 0,131 \mathrm{~g} / \mathrm{cm}^{2}, \mathrm{p}=0,04\right)$. A DMO do colo femoral esteve significativamente reduzida em pacientes com mais de cinco anos de doença, em comparação com as controles saudáveis $(0,710 \pm 0,126$ versus $0,769 \pm 0,130 ; \mathrm{p}=0,02)$. A DMO da coluna lombar diminuiu significativamente em pacientes com menos de 50 anos de idade em comparação com as controles pareadas por idade $(0,958 \pm 0,135$ versus $1,034 \pm 0,125 ; \mathrm{p}=0,03)$. A atividade da doença no momento do estudo não foi associada com redução da DMO ( $p>0,05)$. Foram observadas fraturas anteriores em 18,4\% das pacientes com MII sem associação com a duração da doença e a idade. Altas doses de CE foram associadas a fraturas anteriores em comparação com pacientes sem fraturas $(62,77$ $\pm 42,84$ versus $26,93 \pm 24,31 \mathrm{~g} ; \mathrm{p}=0,05)$. Conclusão: Tais resultados demonstram que a perda óssea é uma complicação importante nas pacientes com MII. A alta freqüência de fraturas observada no presente estudo apóia fortemente medidas preventivas para tais pacientes.

Appenzeller S, Bertolo MB, Costallat LTL: "Frequency of Cognitive Impairment in Rheumatoid Arthritis" (Distúrbios Cognitivos na Artrite Reumatóide). Arthritis Rheum 2003;48(9),S319. Instituição: Disciplina de Reumatologia, Departamento de Clínica Medica, UNICAMP, SP, Brasil.

Objetivo: Determinar a freqüência de distúrbios cognitivos em pacientes com artrite reumatóide (AR). Método: Quarenta pacientes com AR e 40 voluntários sadios, pareados por sexo e idade foram incluídos. A presença de distúrbios cognitivos, ansidade e depressão foi avaliada através do Minimental, teste de memória lógica, teste de memória longa e curta, teste de fluência verbal, teste de atenção, teste de depressão de Beck, BPRS, HAD $\backslash$ CAGE. Foram excluídos pacientes com primeiro grau incompleto e pacientes com menos de 24 pontos no Minimental. Estatística foi realizada através do teste de QuiQuadrado e teste T-Student. Resultados: Não houve diferença significativa entre os dados demográficos dos pacientes e controles. A média de idade dos pacientes foi de 38,25 $(\mathrm{DP}=4,5)$ e dos voluntários sadios de $37,5(\mathrm{DP}=3,1)$ anos.
Distúrbio cognitivo foi observado em 30\% dos pacientes com AR e em 7,5\% dos voluntários sadios. Pacientes com AR obtiveram resultados inferiores aos controles no teste de fluência verbal $(\mathrm{p}<0,05)$, memória lógica $(\mathrm{p}<0,05)$ e memória recente $(p<0,05)$. Não foi observada nenhuma diferença significativa entre os demais testes. Não se observou relação entre a presença de distúrbios cognitivos e a duração da doença, o uso de corticosteróides ou a presença de incapacidade funcional. Conclusão: Observamos uma elevada freqüência de distúrbios cognitivos na AR. A presença de distúrbios cognitivos não esteve associada a manifestações clínicas, ao uso de corticosteróides ou a incapacidade funcional. Mais estudos são necessários para determinar o impacto clínico da presença destas alterações na AR.

Souza SCM, Andrade DCO, Radu AS, Gonçalves CR, Kochen JAL, Borba EF, Laurindo IMM: "Metabolic Syndrome $X$ and Rheumatoid Arthritis in Brazilian Patients" (Síndrome Metabólica X e Artrite Reumatóide em Pacientes Brasileiros). Arthritis Rheum 2003;48(9),S322. Instituição: Divisão de Reumatologia, USP, São Paulo, SP, Brasil. 
Introdução: Estudos epidemiológicos mostraram uma taxa de mortalidade aumentada em conseqüência da doença cardiovascular (DCV) na artrite reumatóide (AR). Por outro lado, a síndrome X é uma doença metabólica presente em ao menos um quarto da população adulta norte-americana, que está associada à aterosclerose acelerada e à morte por DCV. Ambas as doenças estão associadas a níveis elevados de IL-6 e TNF-alfa. Objetivo: Avaliar a prevalência da síndrome X em uma população brasileira com AR e comparar a gravidade da AR em pacientes com ou sem a síndrome X. Métodos: Cinqüenta e cinco pacientes com AR foram selecionados aleatoriamente, $85 \%$ mulheres, com média de idade de $52 \pm 9$ anos e de duração da doença de $15 \pm 8$ anos, $85 \%$ com fator reumatóide positivo. A síndrome $\mathrm{X}$ foi definida pela presença de ao menos três das seguintes anormalidades (critérios ATP III): circunferência da cintura $>102 \mathrm{~cm}$ nos homens e $88 \mathrm{~cm}$ nas mulheres; triglicérides $\geq 150 \mathrm{mg} / \mathrm{dl}(1,69 \mathrm{mmol} / \mathrm{l})$; HDL $<40 \mathrm{mg} / \mathrm{dl}(1,04 \mathrm{mmol} / \mathrm{l})$ nos homens e $<50 \mathrm{mg} / \mathrm{dl}$ $(1,29 \mathrm{mmol} / \mathrm{l})$ nas mulheres; pressão arterial de pelo menos 130/85 mmHg; ou nível sérico de glicose $\geq 110 \mathrm{mg} / \mathrm{dl}$ $(6,1 \mathrm{mmol} / \mathrm{l})$. Foram calculados os escores de predição de risco de IMC e de doença coronariana de Framinghan. Amostras de sangue foram avaliadas para determinar o perfil lipídico completo, a proteína C reativa (PCR) e a velocidade de hemossedimentação. Foram calculados os escores para atividade da doença (DAS28) e HAQ. Resultados: Treze (24\%) pacientes preencheram os critérios para diagnóstico de síndrome X, com uma freqüência similar à da norteamericana. Embora 11 pacientes (20\%) tivessem níveis de triglicérides altos e 23 (42\%) tivessem baixos níveis de HDL, ambas as anormalidades foram encontradas em somente 6 pacientes com AR (11\%), 4 deles com diagnóstico de síndrome X. Quando os pacientes com e sem síndrome X foram comparados, não houve diferença significativa com relação aos parâmetros de AR, tais como positividade de RF, PCR e de velocidade de hemossedimentação, HAQ e DAS28, duração da doença, medicações anteriores e no momento do estudo. A média de IMC dos pacientes com AR foi de $26 \pm 4,2 \mathrm{~kg} / \mathrm{m} 2$, e $29 \%$ foram classificados como grau de obesidade I $(\mathrm{n}=16$, IMC $>26,9)$. Houve correlação significativa entre obesidade e hipertensão $(p=0,016)$, mas não com quaisquer frações de lipoproteínas. $\mathrm{O}$ escore de risco de Framingham (ERF) que avalia o risco de eventos coronarianos em 10 anos esteve na faixa média. Conclusão: A síndrome $\mathrm{X}$ foi detectada em um quarto dos pacientes brasileiros com AR e não teve influência nos parâmetros de atividade da AR, habilidade funcional e respostas terapêuticas apesar dos mecanismos inflamatórios comuns a essas duas doenças.

Erkan $\mathrm{D}^{(1)}$, Sammaritano $\mathrm{L}^{(1)}$, Harrison $\mathrm{MJ}^{(1)}$, Peterson $\mathrm{M}^{(1)}$, Levy RA ${ }^{(2)}$, Yazici $\mathrm{Y}^{(1)}$, Lockshin MD ${ }^{(1)}$ : "APLASA Study: Primary Thrombosis Prevention in Asymptomatic Antiphospholipid Antibody (APL) Positive Patients with Low-dose Aspirin (ASA)" (Estudo APLASA: Prevenção Primária de Trombose em Pacientes Assintomáticos com Anticorpos Antifosfolipídeos [APL] Positivos com Baixa dose de Aspirina [AAS]). Arthritis Rheum 2003;48(9), S358. Instituições: 1. Hospital for Special Surgery, Nova York, NY, EUA; 2. Hospital Universitário Pedro Ernesto, Rio de Janeiro, RJ, Brasil.

A presença de aPL é um fator de risco para tromboses; entretanto, desconhecemos qual paciente com aPL irá desenvolver tromboses. O papel de outros fatores trombogênicos adicionais e de estratégias de prevenção primária não foram profundamente estudados. Objetivo: APLASA é um estudo internacional multicêntrico randomizado e controlado com placebo que foi iniciado em junho de 2001. O objetivo primário é determinar a eficácia de $81 \mathrm{mg}$ diárias de AAS na prevenção primária de tromboses em indivíduos assintomáticos (sem passado de história vascular ou eventos gestacionais) com aPL positivo em um período de 3 anos.
Os objetivos secundários são determinar: a) a incidência de eventos trombóticos em pacientes tratados ou não com AAS; b) a incidência geral de manifestações provavelmente relacionadas com SAF (trombocitopenia, anemia hemolítica, livedo reticularis e enxaqueca); e c) as características clínicas e demográficas de pacientes que desenvolverem eventos vasculares comparados com aqueles que não desenvolverem. Métodos: Todos os pacientes com aPL positivo e assintomáticos (LAC positivo e/ou aCL IgG/M/A $\geq 20$ UI) foram randomizados para receber AAS $81 \mathrm{mg}$ ou placebo diariamente. Os principais critérios de exclusão são história 
de eventos vasculares e gestacionais descritos no critério de Sapporo, ataques isquêmicos transitórios e contra-indicação ao uso de AAS. Os pacientes serão avaliados a cada quadrimestre. Em um subestudo observacional em separado, os pacientes que recusarem a randomização ou que já estejam em uso de AAS serão acompanhados e analisados prospectivamente. Resultados: Por se tratar de um estudo em andamento, as características clínicas e demográficas dos pacientes incluídos até agora serão apresentadas, assim como a relação de eventos. Até 15 de abril de 2003, 52 pacientes foram randomizados para o estudo APLASA. Como parte do subestudo observacional, 41 pacientes consentiram em serem seguidos prospectivamente: $33 \mathrm{com}$ AAS (Obs-AAS) e 8 sem (Obs-sem AAS). Dos 52 pacientes do APLASA, um faleceu de causa não relacionada com SAF (codificado para receber ASA) e 7 foram perdidos do acompanhamento ou desistiram de participar. Nenhum paciente desenvolveu trombose. Dos 33 pacientes do grupo Obs-AAS, um paciente com história de alterações na sustância branca desenvolveu um acidente vascular cerebral isquêmico.

Conclusão: Embora o estudo APLASA esteja em andamento, recrutamos 44,4 pacientes-ano de acompanhamento e não observamos nenhuma trombose. Este é um dos poucos estudos randomizados em indivíduos assintomáticos com anticorpos antifosfolpídeos que irá prover informações valiosas quanto ao curso clínico e conduta nesses pacientes.

\begin{tabular}{lccc}
\hline & $\begin{array}{c}\text { APLASA } \\
\mathbf{n}=52\end{array}$ & $\begin{array}{c}\text { Obs-AAS } \\
\mathbf{n = 3 3}\end{array}$ & $\begin{array}{c}\text { 0bs sem-AAS } \\
\mathbf{n}=8\end{array}$ \\
\hline Idade média na 1. ${ }^{\text {e entrevista }}$ & $44,8 \pm 14,3$ & $45,2 \pm 14,5$ & $45,9 \pm 12,5$ \\
Mulheres / Homens & $48 / 4$ & $32 / 1$ & 27 \\
* LAC (+) e/ou aCL $\geq 40 \mathrm{GPL}$ & 32 & 6 & 5 \\
* aCL 20-39 GPL & 20 & 22,3 & 3 \\
Tempo total de acompanhamento (paciente-ano) & 44,4 & 7,2 \\
\hline
\end{tabular}

Pippa MGB, Latorre MRO, Zerbini CFA: "The Influence of Body Composition on Bone Mineral Density is distinct in BrazilianWomen" (A Influência da Composição Corpórea na Densidade Mineral Óssea É Distinta em Mulheres Brasileiras). Arthritis Rheum 2003;48(9),S493. Instituições: USP; Escola de Saúde Pública da USP; Departamento de Reumatologia do Hospital Heliópolis, São Paulo, SP, Brasil.

Introdução: A densidade mineral óssea (DMO) é influenciada pela composição corpórea (CC), particularmente pelas massas gorda e magra, apresentando valores variáveis dependentes da raça. Objetivos: Levando em conta que a população brasileira é composta por uma ampla variedade de raças, principalmente negros, brancos e pardos, este estudo tem como objetivo avaliar a influência da composição corpórea na DMO de mulheres brasileiras, segundo status de menopausa e raça. Material e Métodos: Foram estudadas 162 mulheres brasileiras (90 brancas e 72 não brancas) com idade entre 20 e 79 anos. A densitometria óssea de dupla emissão com fonte de raios $\mathrm{X}$ foi usada para medir a DMO total (DMOT), a DMO da coluna lombar (DMOCL), a DMO do colo do fêmur (DMOCF), a massa gorda total (MGT) e a massa magra total (MMT). As variáveis foram avaliadas de forma descritiva utilizando média, desvios-padrão, mediana, e valores mínimo e máximo. Na comparação das médias DMO total, DMOs regionais e das medidas de composição corpórea segundo status de menopausa e raça, foi utilizada a análise de variância a um fator. Nas comparações múltiplas utilizamos o teste de Tukey-HSD. A diferença de média entre as variáveis das mulheres brancas e não brancas foi realizada através do teste T-Student. A homogeneidade de variância foi avaliada pelo teste de Lavene e a aderência à curva normal pelo teste de Kolmogorov Smirnov. A correlação entre as variáveis foi estimada utilizando o coeficiente de Pearson. A análise de regressão linear múltipla foi realizada para analisar o efeito conjunto das correlações significativas encontradas. Resultados: 1) Não houve diferenças significativas na comparação das médias da DMO analisadas na coluna lombar e região proximal do fêmur entre as 162 mulheres brancas e não brancas; 2) a média da DMOT 
nas mulheres não brancas na pré menopausa foi maior que a média nas mulheres brancas neste mesmo grupo; 3) nas mulheres brancas pré-menopausa o IMC foi o maior determinante da DMO em todos os sítios estudados; 4) nas mulheres não brancas pré-menopausa a massa magra foi o maior determinante das DMOT e DMOCL, enquanto na DMOCF foi a idade; 5) a massa gorda foi um importante determinante da DMO das mulheres brancas na pósmenopausa em todas as regiões estudadas; 6) nas mulheres não brancas na pós-menopausa a massa magra foi o determinante significativo nas DMO total e regional. Conclusão: Os resultados confirmam os importantes efeitos positivos da massa gorda e massa magra sobre a DMO. Nas mulheres não brancas o achado do importante efeito determinante da massa magra sobre a DMO total e regional, independente do status de menopausa, sugere que medidas que possam preservar e/ou aumentar essa massa devam ser aplicadas em programas para prevenção de osteoporose. Lembramos que o povo brasileiro descende de uma mistura de raças, não apresentando, portanto, características raciais muito bem definidas. A diversidade étnica, representada por diferentes hábitos culturais e nutricionais, é outro dado a ser considerado. Acreditamos que a variabilidade racial e étnica da população brasileira tenham influenciado os resultados.

Restituti R, Castro CHM, Pinheiro MM, Szejnfeld VL: "Awareness, Knowledge, Risk Factors and Current Treatment of Osteoporosis in a Brazilian Cohort of Elderly Subjects" (Consciência, Conhecimento, Fatores de Risco e Tratamento Atual da Osteoporose em uma Coorte Brasileira com Pacientes Idosos). Arthritis Rheum 2003;48(9),S494. Instituição: UNIFESP, SP, Brasil.

Avaliamos a consciência, o conhecimento, os fatores de risco e o tratamento atual de osteoporose em 54 idosos que participaram de um programa social/educacional da terceira idade e 32 idosos residentes num asilo em São Paulo, SP, Brasil. Nosso objetivo foi determinar diferenças entre os dois grupos e detectar diferenças entre homens e mulheres. A população incluiu 70 mulheres e 16 homens, com média de idade de 76 anos. Todos os participantes responderam um questionário aplicado por entrevistador com relação ao conhecimento do que seria a osteoporose, seus fatores de risco e tratamento. A maioria dos idosos residentes no asilo (44\%) tinha nível escolar secundário (Ensino Médio), enquanto a maioria dos que participaram do programa educacional $(55,5 \%)$ não havia completado o primário. Noventa e seis por cento dos idosos conheciam a osteoporose e 49\% definiram-na corretamente. Apesar dos diferentes históricos escolares, o conhecimento sobre a osteoporose e sua correta definição não foram diferentes entre os dois grupos, nem entre homens e mulheres. A televisão, os amigos e os médicos foram identificados como as principais fontes de informação, especialmente entre as mulheres. A maioria dos participantes deste estudo (78\% e 79\% respectivamente) estava ciente que a osteoporose poderia afetar também os homens e que o controle alimentar seria um fator importante. No conjunto, $80 \%$ sabiam que a osteoporose poderia ser prevenida, porém tal consciência foi menor no grupo de pacientes do asilo $(\mathrm{p}<0,001)$. As mulheres acreditavam que poderiam ter mais chance de apresentar osteoporose que os homens $(\mathrm{p}<0,001)$ e, na realidade, um diagnóstico prévio de osteoporose foi mais prevalente entre elas (40\%) quando comparadas aos homens $(6 \% ; \mathrm{p}<0,05)$. Além da idade avançada e da deficiência de estrógeno associada à menopausa, os fatores de risco mais prevalentes incluíram história familiar de fraturas (19\%), o fato de ter fumado no passado $(30 \%)$ e fraturas anteriores (13\%). História familiar de fraturas e fraturas anteriores foram ambas mais comuns entre os idosos que participaram do programa educacional que entre os residentes no asilo $(\mathrm{p}<0,05)$ e entre as mulheres em comparação aos homens $(\mathrm{p}<0,05)$. Somente $25 \%$ dos entrevistados estavam em tratamento específico para osteoporose e em uso de suplementos de cálcio. A utilização de tais suplementos foi mais freqüente entre as mulheres $(30 \%)$ que nos homens $(6 \% ; p<0,01)$. Os resultados demonstram que a maioria dos idosos em uma coorte brasileira tem conhecimento e definição precisa de osteoporose. O estudo também mostra que programas educacionais podem fornecer informações específicas para idosos, influenciando no tratamento da osteoporose. Terapia específica e medidas preventivas para osteoporose foram inapropriadamente baixas para este grupo de idosos, que possui alto risco de osteoporose. 
Pivato FCMM, Sallum AME, Paim LB, Leone C, Liphaus BL, Silva CAA: "Risk Factors of Calcinosis in Juvenile Dermatomyositis (JDM)" (Fatores Associados à Calcinose na Dermatomiosite Juvenil [DMJ]). Arthritis Rheum 2003;48(9),S513. Instituição: Unidade de Reumatologia Pediátrica - ICr-HC-FMUSP.

A calcinose da dermatomiosite juvenil (DMJ) apresenta etiologia desconhecida e está associada aos casos graves, às doses inadequadas e ao retardo no uso de corticosteróide nas fases iniciais da doença. Objetivos: Avaliar as características clínicas, laboratoriais e terapêuticas associadas à calcinose em pacientes com DMJ. Pacientes e métodos: Cinqüenta e quatro pacientes com DMJ foram avaliados retrospectivamente. Todos preencheram critérios de Bohan e Peter para DMJ. Os pacientes com polimiosite foram excluídos. Os dados demográficos, características clínicas: grau de força muscular (I a V do Medical Research Council), pneumonite intersticial (distúrbio ventilatório restritivo), refluxo gastro-esofágico e comprometimento cardíaco (pericardite e/ou miocardite); elevação das enzimas musculares e tratamento (corticoterapia e/ou cloroquina e/ou imunossupressor) foram avaliados. Os pacientes foram distribuídos em dois grupos, de acordo com a presença ou não de calcinose. Análise estatística foi realizada com o teste do qui-quadrado (corrigido para Yates), teste exato de Fisher, T-Student e teste t não pareado. O nível de significância foi de 5\%. Resultados: Calcinose esteve presente em $23(43 \%)$ pacientes, sendo em 6 antes do diagnóstico e em 17 após. A média de idade ao diagnóstico foi de 78,7 $\pm 31,2$ meses nos pacientes com calcinose e $88,9 \pm 33,4$ meses nos pacientes sem calcinose $(p=0,25)$. A média do tempo de doença até o diagnóstico foi de $12,2 \pm 20,4$ meses nos pacientes com calcinose e 7,7 $\pm 10,6$ meses nos pacientes sem calcinose $(p=0,34)$. A calcinose foi associada ao tratamento agressivo da doença (uso de imunossupressores) $(\mathrm{p}=0,03)$, comprometimento cardíaco $(\mathrm{p}=0,01)$ e pulmonar $(\mathrm{p}=0,02)$. A calcinose não foi associada à idade de início da doença superior a 78 meses (média da idade de início encontrada) $(p=0,25)$, tempo de duração da doença até o diagnóstico superior a 12,2 meses (média do tempo de duração da doença antes do diagnóstico) $(p=0,31)$, sexo feminino $(p=0,18)$, grau de força muscular reduzido (I, II ou III) $(p=1,0)$, elevação da média dos níveis séricos das enzimas musculares (CK, TGO, TGP, DHL) $(\mathrm{p}=0,69 ; \mathrm{p}=0,21 ; \mathrm{p}=0,23$ e $\mathrm{p}=0,29$, respectivamente $)$ e a presença de refluxo gastro-esofágico $(p=0,33)$. Conclusões: O aparecimento da calcinose é freqüente na DMJ, habitualmente na evolução da doença. A calcinose foi associada aos casos mais graves, com uso de imunossupressores, envolvimento cardíaco e pulmonar.

Paz IA, Silva CAA, Marques-Dias MJ: "Sydenham's Chorea Treatment: Double Blind Study With Placebo And Prednisone" (Tratamento da Coréia de Sydenham: Estudo Duplo-Cego, Randomizado com Placebo e Prednisona). Arthritis Rheum 2003;48(9),S517. Instituição: Unidades de Neuropediatria e Reumatologia ICr-HC-FMUSP.

Introdução: A coréia de Sydenham (CS) é uma das manifestações maiores da febre reumática (FR). Estudos anteriores evidenciaram que, possivelmente, anticorpos antiestreptocóccicos apresentem reação cruzada com neurônios nos gânglios da base, resultando num distúrbio do movimento, disfunções cognitivas e emocionais, que podem persistir por até dois anos. O tratamento da CS imunomodulador com corticosteróides tem sido raramente estudado, com cerca de 11 publicações, com estudos abertos e pequenas casuísticas. Objetivos: Comparar a resposta terapêutica da prednisona com placebo (através de um estudo prospectivo, duplo-cego, placebo e randomizado) com relação à duração, intensidade e recidivas da CS. Averiguar se a gravidade do quadro da coréia está associada à presença de outras manifestações da FR, de manifestações psiquiátricas, da elevação dos marcadores de fase aguda (VHS e PCR) e dos marcadores de infecção estreptocóccica. Casuística e métodos: No período de 1998 a 2001, 37 pacientes foram selecionados entre 7 e 15 anos anos de idade com CS na fase aguda. Após serem submetidos à 
avaliação neurológica sistematizada e à avaliação reumatológica, cardiológica e psiquiátrica, todos realizaram as provas de fase aguda, a cultura de orofaringe e a ASLO. Um escore de gravidade da coréia foi realizado, baseado na intensidade dos movimentos coréicos e atividades de vida diária. Este foi determinado seis vezes durante um período de três meses. Os pacientes foram distribuídos, após sorteio, em dois grupos terapêuticos: prednisona $(2 \mathrm{mg} / \mathrm{kg} /$ dia por um mês e posterior regressão por mais um mês) e placebo. O protocolo foi aprovado pelo Comitê de Ética de nosso Hospital. Análise estatística foi realizada com os testes exato de Fisher e qui-quadrado. Em todos os testes estatísticos o nível de significância foi de $5 \%(\mathrm{P}<0,05)$.

Resultados: Os principais resultados estão na Tabela ao lado.

Quatro pacientes (18\%) do grupo prednisona e três (20\%) do grupo placebo apresentaram recidivas entre 5 e 12 meses após o primeiro surto $(\mathrm{p}=0,3)$. A positividade dos marcadores de fase aguda e da ASLO, assim como a presença

\begin{tabular}{lcc}
\hline Coréia & $\begin{array}{c}\text { Prednisona } \\
(\mathbf{n}=\mathbf{2 2})\end{array}$ & $\begin{array}{c}\text { Placebo } \\
(\mathbf{n}=\mathbf{1 5})\end{array}$ \\
\hline Escala de intensidade inicial & 33 & 29,7 \\
Escala de intensidade $4 .^{\text {a }}$ semana & 4,9 & 19,9 * \\
Decréscimo na $4 .^{\text {a }}$ semana (\%) & $85 *$ & 33 \\
Duração da coréia (média em dias) & $54,2 \pm 24,7$ & $119,8 \pm 120$ * \\
Uso do haloperidol (n. ${ }^{\circ}$ de pacientes) & 1 & $5{ }^{*}$ \\
\hline * $p<0,001$ & &
\end{tabular}

de cardite, artrite e as manifestações psiquiátricas (sintomas e transtornos obsessivos-compulsivos) não se correlacionaram à intensidade e duração da coréia. Os pacientes não apresentaram eventos adversos importantes associados à prednisona, com exceção do aumento de peso no primeiro mês de uso. Conclusões: $\mathrm{O}$ uso da prednisona em dose imunossupressora reduziu a intensidade e a duração do quadro da coréia quando comparado ao grupo placebo. A prednisona também foi bem tolerada pelos pacientes.

Kiss $\mathrm{MH}^{(1)}$, Reiff $\mathrm{AA}^{(2)}$, Reicin $\mathrm{AS}^{(3)}$, Rose $\mathrm{ES}^{(3)}$, Chen PY(3), Bolognese $\mathrm{JA}^{(3)}$, Adelsberg JV(3): "Rofecoxib Demonstrates Efficacy and Tolerability In Children and Adolescents (Ages 2-17 Years) with Juvenile Rheumatoid Arthritis (JRA) in a 12-Week Randomized Study" (Rofecoxib Demonstra Eficácia e Tolerabilidade em Crianças e Adolescentes [com Idade entre 2 e 17 Anos] com Artrite Reumatóide Juvenil [ARJ] em um Estudo Randomizado de 12 Semanas). Arthritis Rheum 2003;48(9),S650. Instituições: 1. Ambulatório do Hospital das Clínicas da FMUSP, São Paulo, SP, Brasil; 2. Children's Hospital Division of Rheumatology, Los Angeles, CA, EUA; 3. Merck Research Laboratories, Rahway, NJ.

Objetivo: Antiinflamatórios não-seletivos são utilizados para tratar sintomas de ARJ. Este é o primeiro estudo a avaliar a eficácia, a segurança e a tolerabilidade do rofecoxib (ROF), um inibidor seletivo da COX-2, na ARJ. Foi realizado um estudo multinacional de 12 semanas em pacientes de 2 a 17 anos com ARJ para comparar os efeitos de duas doses de ROF (baixa dose de ROF [LD_Rof] $=0,3 \mathrm{mg} /$ $\mathrm{kg} /$ dia, máximo de $12,5 \mathrm{mg} /$ diariamente, e alta dose de ROF [HD_Rof] $=0,6 \mathrm{mg} / \mathrm{kg} /$ dia, máximo de $25 \mathrm{mg} /$ diariamente) e naproxeno (NAP) $7,5 \mathrm{mg} / \mathrm{kg}$ duas vezes por dia. Métodos: Os pacientes foram randomizados em uma proporção 1:1:1 para os grupos LD_Rof, HD_Rof ou NAP. Os pacientes de 2 a 11 anos de idade receberam drogas em forma de suspensão oral, e aqueles entre 12 e 17 anos de idade receberam comprimidos. A hipótese principal era que a proporção de pacientes que atingiam os critérios de ARJ30 (compostos pela Avaliação de Pais/Pacientes do Bem-Estar Geral; Avaliação Global da Atividade da Doença pelo Investigador; Habilidade Funcional [CHAQ]; Número de articulações ativas com artrite; Número de articulações com faixa limitada de movimentos; e VHS) seria similar entre ROF e NAP, conforme avaliado pelo intervalo de confiança (IC) de 95\% predefinido para a taxa de respondedores JRA30 (ROF versus NAP) inteiramente acima 0,5 ; procedimento step-down foi utilizado para as comparações de cada uma das duas doses de rofecoxib versus naproxeno. A taxa de respondedores foi avaliada pelo teste de Mantel-Haenszel. O monitoramento seguro incluiu exames clínicos e laboratoriais e experiências adversas (EAs). Resultados: Foram randomizados 310 pacientes com idade entre 2 e 17 anos (181 < 11 anos) 109, 100 e 101 nos grupos LD_Rof, HD_Rof e NAP, respectivamente. A duração média da ARJ foi de 3,7 
$(\mathrm{SD}=3,32)$ anos, na faixa de 0 a 15 anos de idade. As taxas de respondedores JRA30 foram 46,2\%, 54,1\% e 55,1\% nos grupos LD_Rof, HD_Rof e NAP, respectivamente. Ambas as doses de ROF atingiram os limites pré-especificados para não-inferioridade versus NAP; LD_Rof/NAP RR $=0,81$; IC $95 \%(0,61 ; 1,07)$ e HD_Rof/NAP RR $=0,97$ : IC 95\% $(0,75 ; 1,25)$. No entanto, pareceu haver uma tendência para maior porcentagem de resposta com HD_Rof em comparação com LD_Rof. Tal fato ficou especialmente evidente em pacientes com idade entre 12 e 17 anos. Todos os tratamentos foram bem tolerados por até 12 semanas. Incidências globais de EAs, incluindo EAs sérios, e descontinuações para quaisquer EAs foram geralmente similares entre todos os grupos. A incidência de distúrbios gastrointestinais (GI) foi de 27\%, 32\% e 40\% nos grupos LD_Rof, HD_Rof e NAP, respectivamente; LD_Rof versus NAP foi significativa. Conclusões: Rofecoxib e naproxeno demonstraram eficácia similar no tratamento de ARJ em pacientes com idade entre 2 e 17 anos; foi observada maior eficácia para doses mais altas de ROF em comparação com doses menores dessa mesma substância, especialmente em pacientes com idade entre 12 e 17 anos. O rofecoxib até 12 semanas foi geralmente bem tolerado por crianças e adolescentes com ARJ. Houve EAs gastrointestinais significativamente menores em pacientes que tomaram doses mais baixas de rofecoxib em relação ao naproxeno. 